\title{
Integrability of classical strings dual for noncommutative gauge theories
}

\author{
Takuya Matsumoto $^{a}$ and Kentaroh Yoshida ${ }^{b}$ \\ ${ }^{a}$ Institute for Theoretical Physics and Spinoza Institute, Utrecht University, \\ Leuvenlaan 4, 3854 CE Utrecht, The Netherlands \\ ${ }^{b}$ Department of Physics, Kyoto University, \\ Kyoto 606-8502, Japan \\ E-mail: t.matsumoto@uu.nl, kyoshida@gauge.scphys.kyoto-u.ac.jp
}

ABSTRACT: We derive the gravity duals of noncommutative gauge theories from the YangBaxter sigma model description of the $\mathrm{AdS}_{5} \times \mathrm{S}^{5}$ superstring with classical $r$-matrices. The corresponding classical $r$-matrices are 1) solutions of the classical Yang-Baxter equation (CYBE), 2) skew-symmetric, 3) nilpotent and 4) abelian. Hence these should be called abelian Jordanian deformations. As a result, the gravity duals are shown to be integrable deformations of $\mathrm{AdS}_{5} \times \mathrm{S}^{5}$. Then, abelian twists of $\mathrm{AdS}_{5}$ are also investigated. These results provide a support for the gravity/CYBE correspondence proposed in arXiv:1404.1838.

KEywords: AdS-CFT Correspondence, Sigma Models, Integrable Field Theories, NonCommutative Geometry

ARXIV EPRINT: 1404.3657 


\section{Contents}

1 Introduction 1

2 Integrable deformations of the $\mathrm{AdS}_{5} \times \mathrm{S}^{5}$ superstring 2

2.1 Deforming the $\mathrm{AdS}_{5} \times \mathrm{S}^{5}$ superstring action with CYBE 2

2.2 A classification of classical $r$-matrices 3

3 Examples - gravity duals of NC gauge theories 4

4 Abelian twists of $\mathrm{AdS}_{5} \quad 6$

$\begin{array}{llr}5 & \text { Conclusion and discussion } & 8\end{array}$

$\begin{array}{lr}\text { A Notation and convention } & 8\end{array}$

B Multi-parameter deformations of $\mathrm{AdS}_{5} \quad 9$

$\begin{array}{lll}\text { C Three-parameter abelian twists of } \mathrm{AdS}_{5} & 10\end{array}$

\section{Introduction}

A particular class of gauge/gravity dualities can be seen as deformations of AdS/CFT [1]. With great progress, an integrable structure inhabiting AdS/CFT is well recognized now [2]. The Green-Schwarz string action on $\mathrm{AdS}_{5} \times \mathrm{S}^{5}$ is constructed from a supercoset [3]

$$
\operatorname{PSU}(2,2 \mid 4) /[\mathrm{SO}(1,4) \times \mathrm{SO}(5)]
$$

and the classical integrability follows from the $\mathbb{Z}_{4}$-grading [4]. ${ }^{1}$ Some deformations of the AdS/CFT correspondence may preserve the integrability and hence it would be interesting to consider a method to classify the integrable deformations.

A possible way is to employ the Yang-Baxter sigma model description [11-15] (for $q$-deformed $\mathrm{su}(2)$ and its affine extension, see $[16,17]$ and $[18,19]$, respectively). It has been applied to the $\mathrm{AdS}_{5} \times \mathrm{S}^{5}$ superstring in [20]. According to this approach, integrable deformations of $\mathrm{AdS}_{5} \times \mathrm{S}^{5}$ are given in terms of classical $r$-matrices satisfying modified classical Yang-Baxter equation (mCYBE). The case of [20] corresponds to the classical $r$-matrix of Drinfeld-Jimbo type [21-23]. The metric in the string frame and NS-NS twoform are obtained [24] and some generalizations to other cases are discussed in [25]. It is an intriguing issue to look for the complete gravitational solution. A mirror TBA is also proposed [26].

\footnotetext{
${ }^{1}$ For another formulation [5] of the $\mathrm{AdS}_{5} \times \mathrm{S}^{5}$ superstring, the classical integrability is argued in [6, 7]. For a classification of integrable supercosets, see [8, 9]. For an argument on non-symmetric cosets, see [10].
} 
As a generalization of the Yang-Baxter sigma model description, one may consider classical Yang-Baxter equation (CYBE) rather than mCYBE. The classical action of the $\mathrm{AdS}_{5} \times \mathrm{S}^{5}$ superstring has been constructed in [27]. The integrable deformations are basically regarded as Drinfeld-Reshetikhin twists [21, 22, 28] including Jordanian twists [29, 30] and abelian twists. Hence one can classify integrable deformations of this kind in terms of classical $r$-matrices. We will refer this picture as to the gravity/CYBE correspondence. The first example is presented in [31]. ${ }^{2}$ As another example, Lunin-Maldacena backgrounds $[33,34]$ have also been derived [35].

In this note, we derive the gravity duals of noncommutative $(\mathrm{NC})$ gauge theories $[36,37]$ from the Yang-Baxter sigma model description of the $\mathrm{AdS}_{5} \times \mathrm{S}^{5}$ superstring with classical $r$-matrices. The corresponding classical $r$-matrices are 1) solutions of CYBE, 2) skewsymmetric, 3) nilpotent and 4) abelian. Hence these should be called abelian Jordanian deformations. As a result, the gravity duals of $\mathrm{NC}$ gauge theories are shown to be integrable deformations of $\mathrm{AdS}_{5} \times \mathrm{S}^{5}$. Then, abelian twists of $\mathrm{AdS}_{5}$ are also investigated. A simple example leads to the solution presented in [38]. These results provide a support for the gravity/CYBE correspondence proposed in [35].

This note is organized as follows. Section 2 gives a short summary of the Yang-Baxter sigma model description of the $\mathrm{AdS}_{5} \times \mathrm{S}^{5}$ superstring with classical $r$-matrices satisfying CYBE. Then we introduce three classes of skew-symmetric solutions of CYBE. A new class of $r$-matrices induces abelian Jordanian deformations. Section 3 presents examples of abelian Jordanian type, which lead to the gravity duals of NC gauge theories. In section 4, we consider a deformation of $\mathrm{AdS}_{5}$ with an abelian $r$-matrix concerned with a TsT transformation of $\mathrm{AdS}_{5}$. Section 5 is devoted to conclusion and discussion. We argue some implications of this result and future directions in studies of the gravity/CYBE correspondence. In appendix A our notation and convention are summarized. Appendix B presents the gravity duals of $\mathrm{NC}$ gauge theories with six deformation parameters. Appendix $\mathrm{C}$ describes the detailed computation of three-parameter abelian twists of $\mathrm{AdS}_{5}$. The resulting geometry is also discussed in [39].

\section{Integrable deformations of the $\mathrm{AdS}_{5} \times \mathrm{S}^{5}$ superstring}

We introduce here integrable deformations of the $\mathrm{AdS}_{5} \times \mathrm{S}^{5}$ superstring based on the YangBaxter sigma model description with CYBE [27]. After giving a short review on the general form of deformed actions, we present three classes of classical $r$-matrices.

\subsection{Deforming the $\mathrm{AdS}_{5} \times \mathrm{S}^{5}$ superstring action with CYBE}

A class of integrable deformations of the $\mathrm{AdS}_{5} \times \mathrm{S}^{5}$ superstring can be described with classical $r$-matrices satisfying CYBE [27]. The deformed action is given by

$$
S=-\frac{1}{4}\left(\gamma^{\alpha \beta}-\epsilon^{\alpha \beta}\right) \int_{-\infty}^{\infty} d \tau \int_{0}^{2 \pi} d \sigma \operatorname{Str}\left(A_{\alpha} d \circ \frac{1}{1-\eta R_{g} \circ d} A_{\beta}\right)
$$

\footnotetext{
${ }^{2}$ The solution is closely related to the one in appendix $\mathrm{C}$ of [32].
} 
where the left-invariant one-form $A_{\alpha}$ is defined as

$$
A_{\alpha} \equiv g^{-1} \partial_{\alpha} g, \quad g \in \mathrm{SU}(2,2 \mid 4) .
$$

Here $\gamma^{\alpha \beta}$ and $\epsilon^{\alpha \beta}$ are the flat metric and the anti-symmetric tensor on the string worldsheet. The operator $R_{g}$ is defined as

$$
R_{g}(X) \equiv g^{-1} R\left(g X g^{-1}\right) g,
$$

where a linear operator $R$ satisfies CYBE rather than mCYBE [20]. The R-operator is related to the tensorial representation of classical $r$-matrix through

$$
\begin{aligned}
R(X) & =\operatorname{Tr}_{2}[r(1 \otimes X)]=\sum_{i}\left(a_{i} \operatorname{Tr}\left(b_{i} X\right)-b_{i} \operatorname{Tr}\left(a_{i} X\right)\right) \\
\text { with } \quad r & =\sum_{i} a_{i} \wedge b_{i} \equiv \sum_{i}\left(a_{i} \otimes b_{i}-b_{i} \otimes a_{i}\right)
\end{aligned}
$$

The operator $d$ is given by the following,

$$
d=P_{1}+2 P_{2}-P_{3},
$$

where $P_{i}(i=0,1,2,3)$ are the projections to the $\mathbb{Z}_{4}$-graded components of $\mathfrak{s u}(2,2 \mid 4)$. $P_{0}, P_{2}$ and $P_{1}, P_{3}$ are the projectors to the bosonic and fermionic generators, respectively. In particular, $P_{0}(\mathfrak{s u}(2,2 \mid 4))$ is nothing but $\mathfrak{s o}(1,4) \oplus \mathfrak{s o}(5)$.

For the action (2.1) with an R-operator satisfying CYBE, the Lax pair has been constructed [27] and the classical integrability is ensured in this sense. The $\kappa$-invariance has been proven as well [27].

\subsection{A classification of classical $r$-matrices}

According to the construction of the deformed string action, one may expect the correspondence between integrable deformations of $\mathrm{AdS}_{5} \times \mathrm{S}^{5}$ and classical $r$-matrices, called the gravity/CYBE correspondence [35]. To study along this direction, it would be valuable to classify some typical class of skew-symmetric solutions of CYBE.

In the following, we will consider three types of classical $r$-matrices: ${ }^{3}$ i) Jordanian, ii) abelian, and iii) abelian Jordanian. In particular, the third class will play a crucial role in the next section.

In order to study deformations of $\mathrm{AdS}_{5}$ later, let us consider the case of $\mathfrak{s u}(2,2)$.

i) Jordanian r-matrix.

The first class is classical $r$-matrices of Jordanian type,

$$
r_{\text {Jor }}=E_{i j} \wedge\left(E_{i i}-E_{j j}\right)-2 \sum_{i<k<j} E_{i k} \wedge E_{k j} \quad(1 \leq i<j \leq 4)
$$

where $\left(E_{i j}\right)_{k l} \equiv \delta_{i k} \delta_{j l}$ are the fundamental representation of $\mathfrak{s u}(2,2)$. The characteristic property of Jordanian type $r$-matrices is the nilpotency. Indeed, we could verify that the associated linear R-operator exhibits $\left(R_{\mathrm{Jor}}\right)^{n}=0$ for $n \geq 3$.

\footnotetext{
${ }^{3}$ There would be other types of $r$-matrices. Here we will concentrate on specific examples for simplicity.
} 
Jordanian deformations of the $\mathrm{AdS}_{5} \times \mathrm{S}^{5}$ superstring are considered in [27]. A simple example of the corresponding type IIB supergravity solution is presented in [31]. Only the $\mathrm{AdS}_{5}$ part is deformed and it contains a three-dimensional Schrödinger spacetime as a subspace. Hence it may be regarded as a generalization of [40-43]. It seems likely that the resulting metric is closely related to a null Melvin twist [32].

ii) Abelian r-matrix.

The second class is abelian $r$-matrices composed of the Cartan generators as follows:

$$
r_{\mathrm{Abe}}=\sum_{1 \leq i<j \leq 3} \mu_{i j}\left(E_{i i}-E_{i+1, i+1}\right) \wedge\left(E_{j j}-E_{j+1, j+1}\right),
$$

where $\mu_{i j}=-\mu_{j i}$ are arbitrary parameters. Since these commute with each other and hence satisfy CYBE obviously. The abelian $r$-matrix is a particular example of the Drinfeld-Reshetikhin twists [21, 22, 28]. Note that abelian $r$-matrices are intrinsic to higher rank cases (rank $\geq 2$ ).

It has been shown in [35] that abelian $r$-matrices lead to $\gamma$-deformed backgrounds [34], which include the Lunin-Maldacena background [33] as a particular case. In section 4, we will consider an abelian twist of $\mathrm{AdS}_{5}$ with a single parameter. The resulting geometry corresponds to the one studied in [38]. For multi-parameter cases, see appendix C.

iii) Abelian Jordanian r-matrix.

The third class is a special subclass of i) Jordanian $r$-matrix. A typical example takes the following form,

$$
r_{\mathrm{AJ}}=\sum_{\substack{i, k=1,2 \\ j, l=3,4}} \nu_{(i j),(k l)} E_{i j} \wedge E_{k l},
$$

with arbitrary parameters $\nu_{(i j),(k l)}=-\nu_{(k l),(i j)}$. Because $E_{i j}(i=1,2, j=3,4)$ are the positive root generators and nilpotent, $\left(r_{\mathrm{AJ}}\right)_{a b}$ and $\left(r_{\mathrm{AJ}}\right)_{c d}$ commute each other. In addition, the square of the associated R-operator already vanishes like,

$$
\left(R_{\mathrm{AJ}}\right)^{2}=0,
$$

in comparison to Jordanian $r$-matrices $(2.6)$ for which $\left(R_{\mathrm{Jor}}\right)^{2} \neq 0$ and $\left(R_{\mathrm{Jor}}\right)^{3}=0$ in general. From the two properties, we refer the $r$-matrices (2.8) as abelian Jordanian $r$-matrices, though they are not composed of Cartan generators.

In the next section, we will show that classical $r$-matrices of abelian Jordanian type correspond to the gravity duals of $\mathrm{NC}$ gauge theories [36, 37].

\section{Examples - gravity duals of NC gauge theories}

Let us consider examples of classical $r$-matrices of abelian Jordanian type. These lead to the gravity duals of $\mathrm{NC}$ gauge theories [36, 37]. Hereafter we will concentrate on the $\mathrm{AdS}_{5}$ part and $\mathrm{S}^{5}$ is not deformed. 
A possible example is given by

$$
r_{\mathrm{AJ}}=\mu p_{2} \wedge p_{3}+\nu p_{0} \wedge p_{1}
$$

where $\mu, \nu$ are deformation parameters. Here $p_{\mu}(\mu=0,1,2,3)$ are the upper triangular matrices defined as

$$
p_{\mu} \equiv \frac{1}{2} \gamma_{\mu}-m_{\mu 5}
$$

For our convention of $\gamma_{\mu}$ and the $\mathfrak{s u}(2,2)$ generators, see appendix A. It should be emphasized that $p_{\mu}$ 's are upper triangular and satisfy the following property:

$$
p_{\mu} p_{\nu}=0 .
$$

Thus the classical $r$-matrix (3.1) is of abelian Jordanian type and trivially satisfies CYBE.

To evaluate the Lagrangian (2.1), let us take the following coset parametrization [31]:

$$
g=\exp \left[p_{0} x^{0}+p_{1} x^{1}+p_{2} x^{2}+p_{3} x^{3}\right] \exp \left[\frac{\gamma_{5}}{2} \log z\right] \in \mathrm{SU}(2,2) / \mathrm{SO}(1,4) .
$$

Then the $\mathrm{AdS}_{5}$ part of (2.1) can be rewritten as

$$
\begin{aligned}
L & =-\frac{1}{2}\left(\gamma^{\alpha \beta}-\epsilon^{\alpha \beta}\right) \operatorname{Tr}\left[A_{\alpha} P_{2}\left(J_{\beta}\right)\right] \\
\text { with } \quad J_{\beta} & \equiv \frac{1}{1-2 \eta\left[R_{\mathrm{AJ}}\right]_{g} \circ P_{2}} A_{\beta} .
\end{aligned}
$$

Here $A_{\alpha}=g^{-1} \partial_{\alpha} g$ is restricted to $\mathfrak{s u}(2,2)$ and the associated R-operator $R_{\mathrm{AJ}}$ with (3.1) is determined by the relation (2.4).

It is convenient to divide the Lagrangian $L$ into two parts like $L=L_{G}+L_{B}$, where $L_{G}$ is the metric part and $L_{B}$ is the coupling to an NS-NS two-form, respectively:

$$
\begin{aligned}
L_{G} & \equiv \frac{1}{2}\left[\operatorname{Tr}\left(A_{\tau} P_{2}\left(J_{\tau}\right)\right)-\operatorname{Tr}\left(A_{\sigma} P_{2}\left(J_{\sigma}\right)\right)\right], \\
L_{B} & \equiv \frac{1}{2}\left[\operatorname{Tr}\left(A_{\tau} P_{2}\left(J_{\sigma}\right)\right)-\operatorname{Tr}\left(A_{\sigma} P_{2}\left(J_{\tau}\right)\right)\right] .
\end{aligned}
$$

To derive the explicit form of $L$, it is sufficient to compute the projected current $P_{2}\left(J_{\alpha}\right)$ rather than $J_{\alpha}$ itself. Hence the computation is reduced to solving the following equation,

$$
\left(1-2 \eta P_{2} \circ\left[R_{\mathrm{AJ}}\right]_{g}\right) P_{2}\left(J_{\alpha}\right)=P_{2}\left(A_{\alpha}\right) .
$$

Note that $P_{2}\left(A_{\alpha}\right)$ is expanded with $\gamma$ matrices as follows:

$$
P_{2}\left(A_{\alpha}\right)=\frac{\partial_{\alpha} x^{0} \gamma_{0}+\partial_{\alpha} x^{1} \gamma_{1}+\partial_{\alpha} x^{2} \gamma_{2}+\partial_{\alpha} x^{3} \gamma_{3}+\partial_{\alpha} z \gamma_{5}}{2 z}
$$

Then, by combining (3.9) with (3.8), $P_{2}\left(J_{\alpha}\right)$ can be obtained as

$$
\begin{aligned}
P_{2}\left(J_{\alpha}\right)= & \frac{z\left(z^{2} \partial_{\alpha} x^{0}+2 \eta \nu \partial_{\alpha} x^{1}\right)}{2\left(z^{4}-4 \eta^{2} \nu^{2}\right)} \gamma_{0}+\frac{z\left(z^{2} \partial_{\alpha} x^{1}+2 \eta \nu \partial_{\alpha} x^{0}\right)}{2\left(z^{4}-4 \eta^{2} \nu^{2}\right)} \gamma_{1} \\
& +\frac{z\left(z^{2} \partial_{\alpha} x^{2}+2 \eta \mu \partial_{\alpha} x^{3}\right)}{2\left(z^{4}+4 \eta^{2} \mu^{2}\right)} \gamma_{2}+\frac{z\left(z^{2} \partial_{\alpha} x^{3}-2 \eta \mu \partial_{\alpha} x^{2}\right)}{2\left(z^{4}+4 \eta^{2} \mu^{2}\right)} \gamma_{3}+\frac{\partial_{\alpha} z}{2 z} \gamma_{5} .
\end{aligned}
$$


The resulting forms of $L_{G}$ and $L_{B}$ are given by, respectively,

$$
\begin{aligned}
& L_{G}=-\frac{\gamma^{\alpha \beta}}{2}\left[\frac{z^{2}\left(-\partial_{\alpha} x^{0} \partial_{\beta} x^{0}+\partial_{\alpha} x^{1} \partial_{\beta} x^{1}\right)}{z^{4}-4 \eta^{2} \nu^{2}}+\frac{z^{2}\left(\partial_{\alpha} x^{2} \partial_{\beta} x^{2}+\partial_{\alpha} x^{3} \partial_{\beta} x^{3}\right)}{z^{4}+4 \eta^{2} \mu^{2}}+\frac{\partial_{\alpha} z \partial_{\beta} z}{z^{2}}\right], \\
& L_{B}=\epsilon^{\alpha \beta}\left[-\frac{2 \eta \nu}{z^{4}-4 \eta^{2} \nu^{2}} \partial_{\alpha} x^{0} \partial_{\beta} x^{1}+\frac{2 \eta \mu}{z^{4}+4 \eta^{2} \mu^{2}} \partial_{\alpha} x^{2} \partial_{\beta} x^{3}\right] .
\end{aligned}
$$

Here two deformation parameters $\mu, \nu$ and one normalization factor $\eta$ are contained.

It is easy to see the metric and the NS-NS two-form from (3.11) and (3.12). By introducing new parameter $a$ and $a^{\prime}$ through the identification,

$$
2 \eta \mu=a^{2}, \quad 2 \eta \nu=i a^{\prime 2},
$$

one can find that the resulting metric and two-form exactly agree with the ones of the gravity duals of $\mathrm{NC}$ gauge theories presented in [36, 37], up to the coordinate change $z=1 / u$ and the Wick rotation $x^{0} \rightarrow i x^{0}$. This result shows that the gravity duals of NC gauge theories $[36,37]$ are integrable deformation of $\mathrm{AdS}_{5}$.

\section{Abelian twists of $\mathrm{AdS}_{5}$}

As another kind of integrable deformation of $\mathrm{AdS}_{5}$, we consider an abelian twist of $\mathrm{AdS}_{5}$ with a single parameter. ${ }^{4}$ The resulting geometry corresponds to the one studied in [38]. For a three-parameter generalization, see appendix C.

Let us consider an abelian $r$-matrix,

$$
r_{\text {Abe }}^{(\mu)}=\mu h_{1} \wedge h_{2},
$$

with a deformation parameter $\mu$. Here $h_{i}(i=1,2)$ are two of the Cartan generators of $\mathfrak{s u}(2,2)$ and belong to the fundamental representation,

$$
h_{1}=\operatorname{diag}(-1,1,-1,1), \quad h_{2}=\operatorname{diag}(-1,1,1,-1) .
$$

Then, the $\mathrm{AdS}_{5}$ part of the Lagrangian (2.1) is given by

$$
\begin{aligned}
L & =L_{G}+L_{B}=-\frac{1}{2}\left(\gamma^{\alpha \beta}-\epsilon^{\alpha \beta}\right) \operatorname{Tr}\left[A_{\alpha} P_{2}\left(J_{\beta}\right)\right], \\
\text { with } \quad J_{\beta} & \equiv \frac{1}{1-2 \eta\left[R_{\mathrm{Abe}}^{(\mu)}\right]_{g} \circ P_{2}} A_{\beta},
\end{aligned}
$$

where the current $A_{\alpha}$ is $\mathfrak{s u}(2,2)$-valued and the R-operator associated with (4.1) is defined by the rule (2.4).

The projected current $P_{2}\left(J_{\alpha}\right)$ is to be determined by solving the equation,

$$
\left(1-2 \eta P_{2} \circ\left[R_{\mathrm{Abe}}^{(\mu)}\right]_{g}\right) P_{2}\left(J_{\alpha}\right)=P_{2}\left(A_{\alpha}\right) .
$$

\footnotetext{
${ }^{4}$ Abelian twists of $S^{5}$ have been studied in [35], and these lead to three-parameter $\gamma$-deformed $S^{5}[33,34]$.
} 
By using the coset parameterization (C.5), $P_{2}\left(A_{\alpha}\right)$ is expanded with respect to $\gamma$ matrices,

$$
\begin{aligned}
P_{2}\left(A_{\alpha}\right)=\frac{1}{2}[ & -\partial_{\alpha} \rho \gamma_{1}+i \cosh \rho \partial_{\alpha} \psi_{3} \gamma_{5} \\
& \left.\quad-\sinh \rho\left(\cos \zeta \partial_{\alpha} \psi_{1} \gamma_{2}+\partial_{\alpha} \zeta \gamma_{3}-i \sin \zeta \partial_{\alpha} \psi_{2} \gamma_{0}\right)\right]
\end{aligned}
$$

Then, by plugging (4.6) with (4.5), $P_{2}\left(J_{\alpha}\right)$ can be obtained as

$$
P_{2}\left(J_{\alpha}\right)=j_{\alpha}^{0} \gamma_{0}+j_{\alpha}^{1} \gamma_{1}+j_{\alpha}^{2} \gamma_{2}+j_{\alpha}^{3} \gamma_{3}+j_{\alpha}^{5} \gamma_{5},
$$

with the coefficients

$$
\begin{aligned}
j_{\alpha}^{0} & =\frac{i}{2} \frac{\sin \zeta \sinh \rho}{1+16 \eta^{2} \mu^{2} \sin ^{2} 2 \zeta \sinh ^{4} \rho}\left(\partial_{\alpha} \psi_{2}+8 \eta \mu \cos ^{2} \zeta \sinh ^{2} \rho \partial_{\alpha} \psi_{1}\right), \\
j_{\alpha}^{1} & =-\frac{1}{2} \partial_{\alpha} \rho, \\
j_{\alpha}^{2} & =-\frac{1}{2} \frac{\cos \zeta \sinh \rho}{1+16 \eta^{2} \mu^{2} \sin ^{2} 2 \zeta \sinh ^{4} \rho}\left(\partial_{\alpha} \psi_{1}-8 \eta \mu \sin ^{2} \zeta \sinh ^{2} \rho \partial_{\alpha} \psi_{2}\right), \\
j_{\alpha}^{3} & =-\frac{1}{2} \sinh \rho \partial_{\alpha} \zeta, \\
j_{\alpha}^{5} & =\frac{i}{2} \cosh \rho \partial_{\alpha} \psi_{3} .
\end{aligned}
$$

Finally, the resulting expressions of $L_{G}$ and $L_{B}$ are given by, respectively,

$$
\begin{aligned}
& L_{G}=-\frac{\gamma^{\alpha \beta}}{2}[ \sinh ^{2} \rho \partial_{\alpha} \zeta \partial_{\beta} \zeta+\partial_{\alpha} \rho \partial_{\beta} \rho-\cosh ^{2} \rho \partial_{\alpha} \psi_{3} \partial_{\beta} \psi_{3} \\
&\left.+\frac{\sinh ^{2} \rho}{1+\hat{\gamma}^{2} \sin ^{2} \zeta \cos ^{2} \zeta \sinh ^{4} \rho}\left(\cos ^{2} \zeta \partial_{\alpha} \psi_{1} \partial_{\beta} \psi_{1}+\sin ^{2} \zeta \partial_{\alpha} \psi_{2} \partial_{\beta} \psi_{2}\right)\right], \\
& L_{B}=-\epsilon^{\alpha \beta} \frac{\hat{\gamma} \cos ^{2} \zeta \sin ^{2} \zeta \sinh ^{4} \rho}{1+\hat{\gamma}^{2} \cos ^{2} \zeta \sin ^{2} \zeta \sinh ^{4} \rho} \partial_{\alpha} \psi_{1} \partial_{\beta} \psi_{2} .
\end{aligned}
$$

Here a new deformation parameter $\hat{\gamma}$ is defined as

$$
\hat{\gamma} \equiv 8 \eta \mu \text {. }
$$

Now one can read off the metric and NS-NS two-form from (4.9) and (4.10). By performing the coordinate transformation,

$$
\rho_{1}=\cos \zeta \sinh \rho, \quad \rho_{2}=\sin \zeta \sinh \rho, \quad \rho_{3}=i \cosh \rho,
$$

the resulting metric and NS-NS two-form are given by

$$
\begin{aligned}
d s^{2} & =d \rho_{1}^{2}+d \rho_{2}^{2}+d \rho_{3}^{2}+\frac{\rho_{1}^{2} d \psi_{1}^{2}+\rho_{2}^{2} d \psi_{2}^{2}}{1+\hat{\gamma}^{2} \rho_{1}^{2} \rho_{2}^{2}}+\rho_{3}^{2} d \psi_{3}^{2}+d s_{\mathrm{S}_{5}}^{2}, \\
B_{2} & =\frac{\hat{\gamma} \rho_{1}^{2} \rho_{2}^{2}}{1+\hat{\gamma}^{2} \rho_{1}^{2} \rho_{2}^{2}} d \psi_{1} \wedge d \psi_{2} .
\end{aligned}
$$

Here there is a constraint $\sum_{i=1}^{3} \rho_{i}^{2}=-1$.

These expressions are quite similar to a one-parameter $\gamma$-deformed $S^{5}[33,34]$ and thus the solution with the metric (4.13) and the NS-NS two-form (4.14) may be regarded as a single parameter $\gamma$-deformation of $\mathrm{AdS}_{5}$. 


\section{Conclusion and discussion}

We have shown that the gravity duals of $\mathrm{NC}$ gauge theories $[36,37]$ can be derived from the Yang-Baxter sigma model description of the $\mathrm{AdS}_{5} \times \mathrm{S}^{5}$ superstring with classical $r$-matrices. The corresponding classical $r$-matrices are 1) solutions of CYBE, 2) skewsymmetric, 3) nilpotent and 4) abelian. These should be called abelian Jordanian deformations. As a result, the gravity duals are found to be integrable deformations of $\mathrm{AdS}_{5} \times \mathrm{S}^{5}$. Then, abelian twists of $\mathrm{AdS}_{5}$ have also been investigated and leads to the solutions studied in $[38,39]$. These results provide a support for the gravity/CYBE correspondence proposed in $[35]$.

It is remarkable that our result suggests the integrability of $\mathcal{N}=4$ super Yang-Mills (SYM) theory on noncommutative (NC) spaces. Now there are an enormous amount of arguments on the integrability for scattering amplitudes of $\mathcal{N}=4 \mathrm{SYM}$. Integrable deformations of it would be found on NC spaces. Our analysis has revealed a relation between classical $r$-matrices and deformations parameters of $\mathrm{NC}$ spaces. There may be a close connection to deformation quantization of Kontsevich [44]. Thus one may expect a deep mathematical structure behind the correspondence. We hope that our result could shed light on new fundamental aspects of integrable deformations.

\section{Acknowledgments}

We would like to thank Io Kawaguchi for useful discussions and collaborations at the earlier stage. T.M. also thanks Gleb Arutyunov and Riccardo Borsato for useful discussions. T.M. is supported by the Netherlands Organization for Scientific Research (NWO) under the VICI grant 680-47-602. T.M.'s work is also part of the ERC Advanced grant research programme No. 246974, "Supersymmetry: a window to non-perturbative physics" and of the D-ITP consortium, a program of the NWO that is funded by the Dutch Ministry of Education, Culture and Science (OCW).

\section{A Notation and convention}

We shall here summarize our notation and convention, which basically follow [45].

An element of $\mathfrak{s u}(2,2 \mid 4)$ is identified with an $8 \times 8$ supermatrix,

$$
M=\left[\begin{array}{ll}
m & \xi \\
\zeta & n
\end{array}\right]
$$

Here $m$ and $n$ are $4 \times 4$ matrices with Grassmann even elements, while $\xi$ and $\zeta$ are $4 \times 4$ matrices with Grassmann odd elements. These matrices satisfy a reality condition. Then $m$ and $n$ belong to $\mathfrak{s u}(2,2)=\mathfrak{s o}(2,4)$ and $\mathfrak{s u}(4)=\mathfrak{s o}(6)$, respectively. 
We are concerned with deformations of $\mathrm{AdS}_{5}$. An explicit basis of $\mathfrak{s u}(2,2)$ is the following. The $\gamma$ matrices are given by

$$
\begin{array}{rlrl}
\gamma_{1} & =\left[\begin{array}{cccc}
0 & 0 & 0 & -1 \\
0 & 0 & 1 & 0 \\
0 & 1 & 0 & 0 \\
-1 & 0 & 0 & 0
\end{array}\right], & \gamma_{2}=\left[\begin{array}{cccc}
0 & 0 & 0 & i \\
0 & 0 & i & 0 \\
0 & -i & 0 & 0 \\
-i & 0 & 0 & 0
\end{array}\right], \quad \gamma_{3}=\left[\begin{array}{llll}
0 & 0 & 1 & 0 \\
0 & 0 & 0 & 1 \\
1 & 0 & 0 & 0 \\
0 & 1 & 0 & 0
\end{array}\right], \\
\gamma_{0}=\left[\begin{array}{cccc}
0 & 0 & 1 & 0 \\
0 & 0 & 0 & -1 \\
-1 & 0 & 0 & 0 \\
0 & 1 & 0 & 0
\end{array}\right], & \gamma_{5}=i \gamma_{1} \gamma_{2} \gamma_{3} \gamma_{0}=\left[\begin{array}{cccc}
1 & 0 & 0 & 0 \\
0 & 1 & 0 & 0 \\
0 & 0 & -1 & 0 \\
0 & 0 & 0 & -1
\end{array}\right]
\end{array}
$$

and satisfy the Clifford algebra

$$
\left\{\gamma_{\mu}, \gamma_{\nu}\right\}=2 \eta_{\mu \nu}, \quad\left\{\gamma_{\mu}, \gamma_{5}\right\}=0, \quad\left(\gamma_{5}\right)^{2}=1
$$

The Lie algebra $\mathfrak{s o}(1,4)$ is formed by the generators

$$
m_{\mu \nu}=\frac{1}{4}\left[\gamma_{\mu}, \gamma_{\nu}\right], \quad m_{\mu 5}=\frac{1}{4}\left[\gamma_{\mu}, \gamma_{5}\right] \quad(\mu, \nu=0,1,2,3)
$$

and then $\mathfrak{s o}(2,4)=\mathfrak{s u}(2,2)$ is spanned by the following set:

$$
m_{\mu \nu}, \quad m_{\mu 5}, \quad \gamma_{\mu}, \gamma_{5}
$$

\section{B Multi-parameter deformations of $\mathrm{AdS}_{5}$}

We present here multi-parameter deformations of $\mathrm{AdS}_{5}$ by using the Yang-Baxter sigma model description with classical $r$-matrices. These may be regarded as a multi-parameter generalization of the gravity duals of $\mathrm{NC}$ gauge theories discussed in $[36,37]$. In the original construction [36, 37] based on twisted T-dualities, it would be intricate to perform T-dualities many times. A technical advantage of the Yang-Baxter sigma model description is that a single $r$-matrix gives the corresponding metric and NS-NS two-form in a more direct way.

Let us consider the following classical $r$-matrix,

$$
\begin{aligned}
r_{\mathrm{AJ}}= & \mu_{1} p_{2} \wedge p_{3}+\mu_{2} p_{3} \wedge p_{1}+\mu_{3} p_{1} \wedge p_{2} \\
& +\nu_{1} p_{0} \wedge p_{1}+\nu_{2} p_{0} \wedge p_{2}+\nu_{3} p_{0} \wedge p_{3},
\end{aligned}
$$

where $\mu_{1}, \mu_{2}, \mu_{3}$ and $\nu_{1}, \nu_{2}, \nu_{3}$ are six deformation parameters, and $p_{\mu}$ are defined in (3.2). By following the analysis in section 3 , it is straightforward to get the deformed string 
action. For simplicity, we shall write down only the resulting metric and NS-NS two-form,

$$
\begin{aligned}
d s^{2}=\frac{d z^{2}}{z^{2}}+z^{2} G[ & -\left(z^{4}+4 \eta^{2}\left(\mu_{1}^{2}+\mu_{2}^{2}+\mu_{3}^{2}\right)\right)\left(d x^{0}\right)^{2}+\left(z^{4}+4 \eta^{2}\left(\mu_{1}^{2}-\nu_{2}^{2}-\nu_{3}^{2}\right)\right)\left(d x^{1}\right)^{2} \\
& +\left(z^{4}+4 \eta^{2}\left(\mu_{2}^{2}-\nu_{3}^{2}-\nu_{1}^{2}\right)\right)\left(d x^{2}\right)^{2}+\left(z^{4}+4 \eta^{2}\left(\mu_{3}^{2}-\nu_{1}^{2}-\nu_{2}^{2}\right)\right)\left(d x^{3}\right)^{2} \\
& -8 \eta^{2}\left[\left(\mu_{2} \nu_{3}-\mu_{3} \nu_{2}\right) d x^{0} d x^{1}+\left(\mu_{3} \nu_{1}-\mu_{1} \nu_{3}\right) d x^{0} d x^{2}+\left(\mu_{1} \nu_{2}-\mu_{2} \nu_{1}\right) d x^{0} d x^{3}\right. \\
& \left.\left.-\left(\mu_{1} \mu_{2}+\nu_{1} \nu_{2}\right) d x^{1} d x^{2}-\left(\mu_{2} \mu_{3}+\nu_{2} \nu_{3}\right) d x^{2} d x^{3}-\left(\mu_{1} \mu_{3}+\nu_{1} \nu_{3}\right) d x^{1} d x^{3}\right]\right]
\end{aligned}
$$

$$
\begin{aligned}
B_{2}=2 \eta G[ & \left(z^{4} \mu_{1}-\eta^{2} \nu_{1} K\right) d x^{2} \wedge d x^{3}-\left(z^{4} \nu_{1}+\eta^{2} \mu_{1} K\right) d x^{0} \wedge d x^{1} \\
& +\left(z^{4} \mu_{2}-\eta^{2} \nu_{2} K\right) d x^{3} \wedge d x^{1}-\left(z^{4} \nu_{2}+\eta^{2} \mu_{2} K\right) d x^{0} \wedge d x^{2} \\
& \left.+\left(z^{4} \mu_{3}-\eta^{2} \nu_{3} K\right) d x^{1} \wedge d x^{2}-\left(z^{4} \nu_{3}+\eta^{2} \mu_{3} K\right) d x^{0} \wedge d x^{3}\right] .
\end{aligned}
$$

Here a scalar function $G$ and a constant parameter $K$ are defined as

$$
\begin{aligned}
G^{-1} & \equiv z^{8}+4 \eta^{2} z^{4}\left(\mu_{1}^{2}+\mu_{2}^{2}+\mu_{3}^{2}-\nu_{1}^{2}-\nu_{2}^{2}-\nu_{3}^{2}\right)-\eta^{4} K^{2} \\
K & \equiv 4\left(\mu_{1} \nu_{1}+\mu_{2} \nu_{2}+\mu_{3} \nu_{3}\right) .
\end{aligned}
$$

By taking the following identification of the parameters

$$
2 \eta \mu_{1}=a^{2}, \quad 2 \eta \nu_{1}=i a^{\prime 2}, \quad \mu_{2}=\mu_{3}=\nu_{2}=\nu_{3}=0
$$

and performing a Wick rotation $x_{0} \rightarrow i x_{0}$, one can reproduce the metric and NS-NS twoform of the two-parameter case [36, 37].

Although we have discussed only the metric and NS-NS two-form so far, one may expect that these should be complemented with the other field components and gives a complete solution of type IIB supergravity on the basis of the $\kappa$-invariance of the action. It still needs to be justified and it would be an important task. Once it has been justified, it gives a consistent string background because it is basically obtained by performing a chain of (twisted) T-dualities for $\mathrm{AdS}_{5}$.

\section{Three-parameter abelian twists of $\mathrm{AdS}_{5}$}

Let us consider here a three-parameter generalization of the abelian deformation of $\mathrm{AdS}_{5}$ discussed in section 4 .

We will consider the following classical $r$-matrix,

$$
r_{\mathrm{Abe}}^{\left(\mu_{1}, \mu_{2}, \mu_{3}\right)}=\mu_{3} h_{1} \wedge h_{2}+\mu_{1} h_{2} \wedge h_{3}+\mu_{2} h_{3} \wedge h_{1},
$$

with deformation parameters $\mu_{i}$. Here $h_{i}$ are the three Cartan generators of $\mathfrak{s u}(2,2)$ and belong to the fundamental representation,

$$
h_{1}=\operatorname{diag}(-1,1,-1,1), \quad h_{2}=\operatorname{diag}(-1,1,1,-1), \quad h_{3}=\operatorname{diag}(1,1,-1,-1) .
$$


By using the $r$-matrix (C.1), the $\mathrm{AdS}_{5}$ part of (2.1) can be rewritten as

$$
\begin{aligned}
L & =L_{G}+L_{B}=-\frac{1}{2}\left(\gamma^{\alpha \beta}-\epsilon^{\alpha \beta}\right) \operatorname{Tr}\left[A_{\alpha} P_{2}\left(J_{\beta}\right)\right], \\
\text { with } \quad J_{\beta} & \equiv \frac{1}{1-2 \eta\left[R_{\text {Abe }}^{\left(\mu_{1}, \mu_{2}, \mu_{3}\right)}\right]_{g} \circ P_{2}} A_{\beta},
\end{aligned}
$$

where $A_{\alpha}=g^{-1} \partial_{\alpha} g$ is restricted to $\mathfrak{s u}(2,2)$ and the R-operator associated with (C.1) is determined by the rule (2.4).

To evaluate the Lagrangian (C.3), let us adopt the following coset parametrization [24]:

$$
g=\Lambda\left(\psi_{1}, \psi_{2}, \psi_{3}\right) \Xi(\zeta) \check{g}_{\rho}(\rho) \quad \in \mathrm{SU}(2,2) / \mathrm{SO}(1,4)
$$

Here the matrices $\Lambda, \Xi$ and $\check{g}_{\rho}$ are defined as

$$
\begin{aligned}
\Lambda\left(\psi_{1}, \psi_{2}, \psi_{3}\right) \equiv \exp \left[\frac{i}{2}\left(\psi_{1} h_{1}+\psi_{2} h_{2}+\psi_{3} h_{3}\right)\right], \\
\Xi(\zeta) \equiv\left(\begin{array}{cccc}
\cos \frac{\zeta}{2} & \sin \frac{\zeta}{2} & 0 & 0 \\
-\sin \frac{\zeta}{2} & \cos \frac{\zeta}{2} & 0 & 0 \\
0 & 0 & \cos \frac{\zeta}{2} & -\sin \frac{\zeta}{2} \\
0 & 0 & \sin \frac{\zeta}{2} & \cos \frac{\zeta}{2}
\end{array}\right), \\
\check{g}_{\rho}(\rho) \equiv\left(\begin{array}{cccc}
\cosh \frac{\rho}{2} & 0 & 0 & \sinh \frac{\rho}{2} \\
0 & \cosh \frac{\rho}{2} & -\sinh \frac{\rho}{2} & 0 \\
0 & -\sinh \frac{\rho}{2} & \cosh \frac{\rho}{2} & 0 \\
\sinh \frac{\rho}{2} & 0 & 0 & \cosh \frac{\rho}{2}
\end{array}\right) .
\end{aligned}
$$

To find the projected current $P_{2}\left(J_{\alpha}\right)$, it is necessary to solve the following equation,

$$
\left(1-2 \eta P_{2} \circ\left[R_{\mathrm{Abe}}^{\left(\mu_{1}, \mu_{2}, \mu_{3}\right)}\right]_{g}\right) P_{2}\left(J_{\alpha}\right)=P_{2}\left(A_{\alpha}\right) .
$$

Note that $P_{2}\left(A_{\alpha}\right)$ is expanded with respect to the $\gamma$ matrices,

$$
\begin{aligned}
P_{2}\left(A_{\alpha}\right)=\frac{1}{2}[ & -\partial_{\alpha} \rho \gamma_{1}+i \cosh \rho \partial_{\alpha} \psi_{3} \gamma_{5} \\
& \left.\quad-\sinh \rho\left(\cos \zeta \partial_{\alpha} \psi_{1} \gamma_{2}+\partial_{\alpha} \zeta \gamma_{3}-i \sin \zeta \partial_{\alpha} \psi_{2} \gamma_{0}\right)\right]
\end{aligned}
$$

Then, by combining (C.7) with (C.6), $P_{2}\left(J_{\alpha}\right)$ can be obtained as

$$
P_{2}\left(J_{\alpha}\right)=j_{\alpha}^{0} \gamma_{0}+j_{\alpha}^{1} \gamma_{1}+j_{\alpha}^{2} \gamma_{2}+j_{\alpha}^{3} \gamma_{3}+j_{\alpha}^{5} \gamma_{5}
$$


with the coefficients

$$
\begin{aligned}
j_{\alpha}^{0}=- & \frac{i}{2} \frac{\sin \zeta \sinh \rho}{1-16 \eta^{2}\left[\left(\mu_{1}^{2} \sin ^{2} \zeta+\mu_{2}^{2} \cos ^{2} \zeta\right) \sinh ^{2} 2 \rho-\mu_{3}^{2} \sin ^{2} 2 \zeta \sinh ^{4} \rho\right]} \\
\times & {\left[\left(-1+16 \eta^{2} \mu_{2}^{2} \cos ^{2} \zeta \sinh ^{2} 2 \rho\right) \partial_{\alpha} \psi_{2}\right.} \\
& -8 \eta\left(\mu_{1}-8 \eta \mu_{2} \mu_{3} \cos ^{2} \zeta \sinh ^{2} \rho\right) \cosh ^{2} \rho \partial_{\alpha} \psi_{3} \\
& \left.-8 \eta\left(\mu_{3}-8 \eta \mu_{1} \mu_{2} \cosh ^{2} \rho\right) \cos ^{2} \zeta \sinh ^{2} \rho \partial_{\alpha} \psi_{1}\right], \\
j_{\alpha}^{1}=- & \frac{1}{2} \partial_{\alpha} \rho, \\
j_{\alpha}^{2}=\frac{1}{2} & \frac{\cos \zeta \sinh \rho}{1-16 \eta^{2}\left[\left(\mu_{1}^{2} \sin ^{2} \zeta+\mu_{2}^{2} \cos ^{2} \zeta\right) \sinh ^{2} 2 \rho-\mu_{3}^{2} \sin ^{2} 2 \zeta \sinh ^{4} \rho\right]} \\
\times & {\left[\left(-1+16 \eta^{2} \mu_{1}^{2} \sin ^{2} \zeta \sinh ^{2} 2 \rho\right) \partial_{\alpha} \psi_{1}\right.} \\
& +8 \eta\left(\mu_{3}+8 \eta \mu_{1} \mu_{2} \cosh ^{2} \rho\right) \sin ^{2} \zeta \sinh ^{2} \rho \partial_{\alpha} \psi_{2} \\
& \left.+8 \eta\left(\mu_{2}+8 \eta \mu_{1} \mu_{3} \sin ^{2} \zeta \sinh ^{2} \rho\right) \cosh ^{2} \rho \partial_{\alpha} \psi_{3}\right], \\
j_{\alpha}^{3}=- & \frac{1}{2} \sinh \rho \partial_{\alpha} \zeta, \\
j_{\alpha}^{5}=\frac{i}{2} & \frac{\cosh \rho}{1-16 \eta^{2}\left[\left(\mu_{1}^{2} \sin ^{2} \zeta+\mu_{2}^{2} \cos ^{2} \zeta\right) \sinh ^{2} 2 \rho-\mu_{3}^{2} \sin ^{2} 2 \zeta \sinh ^{4} \rho\right]} \\
\times & {\left[\left(1+16 \eta^{2} \mu_{3}^{2} \sin ^{2} 2 \zeta \sinh ^{4} \rho\right) \partial_{\alpha} \psi_{3}\right.} \\
& \quad-8 \eta\left(\mu_{2}-8 \eta \mu_{1} \mu_{3} \sin ^{2} \zeta \sinh ^{2} \rho\right) \cos ^{2} \zeta \sinh ^{2} \rho \partial_{\alpha} \psi_{1} \\
& \left.+8 \eta\left(\mu_{1}+8 \eta \mu_{2} \mu_{3} \cos ^{2} \zeta \sinh ^{2} \rho\right) \sin ^{2} \zeta \sinh ^{2} \rho \partial_{\alpha} \psi_{2}\right] .
\end{aligned}
$$

Finally, $L_{G}$ and $L_{B}$ are given by, respectively,

$$
\begin{aligned}
L_{G}=-\frac{\gamma^{\alpha \beta}}{2}[ & -\sinh ^{2} \rho \partial_{\alpha} \rho \partial_{\beta} \rho \\
& +\left(\sin \zeta \sinh \rho \partial_{\alpha} \zeta-\cos \zeta \cosh \rho \partial_{\alpha} \rho\right)\left(\sin \zeta \sinh \rho \partial_{\beta} \zeta-\cos \zeta \cosh \rho \partial_{\beta} \rho\right) \\
& +\left(\cos \zeta \sinh \rho \partial_{\alpha} \zeta+\sin \zeta \cosh \rho \partial_{\alpha} \rho\right)\left(\cos \zeta \sinh \rho \partial_{\beta} \zeta+\sin \zeta \cosh \rho \partial_{\beta} \rho\right) \\
& +\hat{G}\left[\sinh ^{2} \rho\left(\cos ^{2} \zeta \partial_{\alpha} \psi_{1} \partial_{\beta} \psi_{1}+\sin ^{2} \zeta \partial_{\alpha} \psi_{2} \partial_{\beta} \psi_{2}\right)-\cosh ^{2} \rho \partial_{\alpha} \psi_{3} \partial_{\beta} \psi_{3}\right. \\
& \left.\left.\quad-\cos ^{2} \zeta \sin ^{2} \zeta \cosh ^{2} \rho \sinh ^{2} \rho\left(\sum_{i} \hat{\gamma}_{i} \partial_{\alpha} \psi_{i}\right)\left(\sum_{j} \hat{\gamma}_{j} \partial_{\beta} \psi_{j}\right)\right]\right] \\
L_{B}=-\epsilon^{\alpha \beta} \hat{G}\left[\hat{\gamma}_{3} \cos ^{2} \zeta \sin ^{2} \zeta \sinh ^{4} \rho \partial_{\alpha} \psi_{1} \partial_{\beta} \psi_{2}\right. & \left.\quad-\sinh ^{2} \rho \cosh ^{2} \rho\left(\hat{\gamma}_{2} \cos ^{2} \zeta \partial_{\alpha} \psi_{3} \partial_{\beta} \psi_{1}+\hat{\gamma}_{1} \sin ^{2} \zeta \partial_{\alpha} \psi_{2} \partial_{\beta} \psi_{3}\right)\right] .
\end{aligned}
$$

Here a scalar function $\hat{G}$ is defined as

$$
\hat{G}^{-1} \equiv 1-\left(\hat{\gamma}_{1}^{2} \sin ^{2} \zeta+\hat{\gamma}_{2}^{2} \cos ^{2} \zeta\right) \cosh ^{2} \rho \sinh ^{2} \rho+\hat{\gamma}_{3}^{2} \cos ^{2} \zeta \sin ^{2} \zeta \sinh ^{4} \rho,
$$

and new deformation parameters $\hat{\gamma}_{i}$ are

$$
\hat{\gamma}_{i} \equiv 8 \eta \mu_{i} \quad(i=1,2,3)
$$


By performing the coordinate transformation (4.12), the metric and NS-NS two-form associated with (C.10) and (C.11) are written into a compact forms,

$$
\begin{aligned}
d s^{2} & =\sum_{i=1}^{3}\left(d \rho_{i}^{2}+\hat{G} \rho_{i}^{2} d \psi_{i}^{2}\right)+\hat{G} \rho_{1}^{2} \rho_{2}^{2} \rho_{3}^{2}\left(\sum_{i=1}^{3} \hat{\gamma}_{i} d \psi_{i}\right)^{2}+d s_{\mathrm{S}_{5}}^{2} \\
B_{2} & =\hat{G}\left(\hat{\gamma}_{3} \rho_{1}^{2} \rho_{2}^{2} d \psi_{1} \wedge d \psi_{2}+\hat{\gamma}_{1} \rho_{2}^{2} \rho_{3}^{2} d \psi_{2} \wedge d \psi_{3}+\hat{\gamma}_{2} \rho_{3}^{2} \rho_{1}^{2} d \psi_{3} \wedge d \psi_{1}\right) .
\end{aligned}
$$

Here there is a constraint $\sum_{i=1}^{3} \rho_{i}^{2}=-1$ and $\hat{G}$ turns out to be

$$
\hat{G}^{-1}=1+\hat{\gamma}_{3}^{2} \rho_{1}^{2} \rho_{2}^{2}+\hat{\gamma}_{1}^{2} \rho_{2}^{2} \rho_{3}^{2}+\hat{\gamma}_{2}^{2} \rho_{3}^{2} \rho_{1}^{2} .
$$

These are quite similar to $\gamma$-deformed $\mathrm{S}^{5}[33,34]$ and hence the metric (C.14) and NS-NS two-form (C.15) may be regarded as $\gamma$-deformed $\mathrm{AdS}_{5}$. The same geometry is also derived in an earlier work [39].

The one-parameter result in section 4 is reproduced by setting the parameters as

$$
\hat{\gamma}_{1}=\hat{\gamma}_{2}=0, \quad \hat{\gamma}_{3}=\hat{\gamma}
$$

Open Access. This article is distributed under the terms of the Creative Commons Attribution License (CC-BY 4.0), which permits any use, distribution and reproduction in any medium, provided the original author(s) and source are credited.

\section{References}

[1] J.M. Maldacena, The large- $N$ limit of superconformal field theories and supergravity, Adv. Theor. Math. Phys. 2 (1998) 231 [Int. J. Theor. Phys. 38 (1999) 1113] [hep-th/9711200] [INSPIRE].

[2] N. Beisert et al., Review of AdS/CFT integrability: an overview, Lett. Math. Phys. 99 (2012) 3 [arXiv:1012 .3982] [INSPIRE].

[3] R.R. Metsaev and A.A. Tseytlin, Type IIB superstring action in $A d S_{5} \times S^{5}$ background, Nucl. Phys. B 533 (1998) 109 [hep-th/9805028] [InSPIRE].

[4] I. Bena, J. Polchinski and R. Roiban, Hidden symmetries of the $A d S_{5} \times S^{5}$ superstring, Phys. Rev. D 69 (2004) 046002 [hep-th/0305116] [INSPIRE].

[5] R. Roiban and W. Siegel, Superstrings on $A d S_{5} \times S^{5}$ supertwistor space, JHEP 11 (2000) 024 [hep-th/0010104] [INSPIRE].

[6] M. Hatsuda and K. Yoshida, Classical integrability and super Yangian of superstring on $A d S_{5} \times S^{5}$, Adv. Theor. Math. Phys. 9 (2005) 703 [hep-th/0407044] [INSPIRE].

[7] M. Hatsuda and K. Yoshida, Super Yangian of superstring on $A d S_{5} \times S^{5}$ revisited, Adv. Theor. Math. Phys. 15 (2011) 1485 [arXiv:1107.4673] [inSPIRE].

[8] K. Zarembo, Strings on semisymmetric superspaces, JHEP 05 (2010) 002 [arXiv: 1003.0465] [INSPIRE].

[9] L. Wulff, Superisometries and integrability of superstrings, arXiv:1402.3122 [INSPIRE].

[10] S. Schäfer-Nameki, M. Yamazaki and K. Yoshida, Coset construction for duals of non-relativistic CFTs, JHEP 05 (2009) 038 [arXiv:0903.4245] [INSPIRE]. 
[11] C. Klimčík, Yang-Baxter $\sigma$-models and dS/AdS T duality, JHEP 12 (2002) 051 [hep-th/0210095] [INSPIRE].

[12] C. Klimčík, On integrability of the Yang-Baxter $\sigma$-model, J. Math. Phys. 50 (2009) 043508 [arXiv:0802.3518] [INSPIRE].

[13] C. Klimčík, Integrability of the bi-Yang-Baxter $\sigma$-model, arXiv:1402.2105 [INSPIRE].

[14] R. Squellari, Yang-Baxter $\sigma$ model: quantum aspects, Nucl. Phys. B 881 (2014) 502 [arXiv: 1401.3197] [INSPIRE].

[15] F. Delduc, M. Magro and B. Vicedo, On classical q-deformations of integrable $\sigma$-models, JHEP 11 (2013) 192 [arXiv: 1308.3581] [INSPIRE].

[16] I. Kawaguchi and K. Yoshida, Hybrid classical integrability in squashed $\sigma$-models, Phys. Lett. B 705 (2011) 251 [arXiv:1107.3662] [INSPIRE].

[17] I. Kawaguchi and K. Yoshida, Hybrid classical integrable structure of squashed $\sigma$-models: a short summary, J. Phys. Conf. Ser. 343 (2012) 012055 [arXiv:1110.6748] [InSPIRE].

[18] I. Kawaguchi, T. Matsumoto and K. Yoshida, The classical origin of quantum affine algebra in squashed $\sigma$-models, JHEP 04 (2012) 115 [arXiv:1201.3058] [INSPIRE].

[19] I. Kawaguchi, T. Matsumoto and K. Yoshida, On the classical equivalence of monodromy matrices in squashed $\sigma$-model, JHEP 06 (2012) 082 [arXiv: 1203.3400] [INSPIRE].

[20] F. Delduc, M. Magro and B. Vicedo, An integrable deformation of the $A d S_{5} \times S^{5}$ superstring action, Phys. Rev. Lett. 112 (2014) 051601 [arXiv: 1309.5850] [INSPIRE].

[21] V.G. Drinfeld́, Hopf algebras and the quantum Yang-Baxter equation, Sov. Math. Dokl. 32 (1985) 254 [INSPIRE].

[22] V.G. Drinfel'd, Quantum groups, J. Sov. Math. 41 (1988) 898 [Zap. Nauchn. Semin. 155 (1986) 18] [INSPIRE].

[23] M. Jimbo, A q difference analog of $\mathrm{U}(g)$ and the Yang-Baxter equation, Lett. Math. Phys. 10 (1985) 63 [inSPIRE].

[24] G. Arutyunov, R. Borsato and S. Frolov, S-matrix for strings on $\eta$-deformed $A d S_{5} \times S^{5}$, JHEP 04 (2014) 002 [arXiv: 1312.3542] [INSPIRE].

[25] B. Hoare, R. Roiban and A.A. Tseytlin, On deformations of $A d S_{n} \times S^{n}$ supercosets, arXiv: 1403.5517 [INSPIRE].

[26] G. Arutyunov, M. de Leeuw and S.J. van Tongeren, On the exact spectrum and mirror duality of the $\left(A d S_{5} \times S^{5}\right)_{\eta}$ superstring, arXiv:1403.6104 [INSPIRE].

[27] I. Kawaguchi, T. Matsumoto and K. Yoshida, Jordanian deformations of the $A d S_{5} \times S^{5}$ superstring, JHEP 04 (2014) 153 [arXiv:1401.4855] [INSPIRE].

[28] N. Reshetikhin, Multiparameter quantum groups and twisted quasitriangular Hopf algebras, Lett. Math. Phys. 20 (1990) 331 [inSPIRE].

[29] A. Stolin and P.P. Kulish, New rational solutions of Yang-Baxter equation and deformed Yangians, Czech. J. Phys. 47 (1997) 123 [q-alg/9608011].

[30] P.P. Kulish, V.D. Lyakhovsky and A.I. Mudrov, Extended Jordanian twists for Lie algebras, J. Math. Phys. 40 (1999) 4569 [math.QA/9806014] [InSPIRE].

[31] I. Kawaguchi, T. Matsumoto and K. Yoshida, A Jordanian deformation of AdS space in type IIB supergravity, arXiv:1402.6147 [INSPIRE]. 
[32] V.E. Hubeny, M. Rangamani and S.F. Ross, Causal structures and holography, JHEP 07 (2005) 037 [hep-th/0504034] [INSPIRE].

[33] O. Lunin and J.M. Maldacena, Deforming field theories with $\mathrm{U}(1) \times \mathrm{U}(1)$ global symmetry and their gravity duals, JHEP 05 (2005) 033 [hep-th/0502086] [INSPIRE].

[34] S. Frolov, Lax pair for strings in Lunin-Maldacena background, JHEP 05 (2005) 069 [hep-th/0503201] [INSPIRE].

[35] T. Matsumoto and K. Yoshida, Lunin-Maldacena backgrounds from the classical Yang-Baxter equation - towards the gravity/CYBE correspondence, arXiv:1404.1838 [INSPIRE].

[36] A. Hashimoto and N. Itzhaki, Noncommutative Yang-Mills and the AdS/CFT correspondence, Phys. Lett. B 465 (1999) 142 [hep-th/9907166] [INSPIRE].

[37] J.M. Maldacena and J.G. Russo, Large- $N$ limit of noncommutative gauge theories, JHEP 09 (1999) 025 [hep-th/9908134] [INSPIRE].

[38] D. Dhokarh, S.S. Haque and A. Hashimoto, Melvin twists of global $A d S_{5} \times S^{5}$ and their non-commutative field theory dual, JHEP 08 (2008) 084 [arXiv:0801.3812] [INSPIRE].

[39] T. McLoughlin and I. Swanson, Integrable twists in AdS/CFT, JHEP 08 (2006) 084 [hep-th/0605018] [INSPIRE].

[40] I. Kawaguchi and K. Yoshida, Classical integrability of Schrödinger $\sigma$-models and q-deformed Poincaré symmetry, JHEP 11 (2011) 094 [arXiv: 1109.0872] [INSPIRE].

[41] I. Kawaguchi and K. Yoshida, Exotic symmetry and monodromy equivalence in Schrödinger $\sigma$-models, JHEP 02 (2013) 024 [arXiv: 1209.4147] [inSPIRE].

[42] I. Kawaguchi, T. Matsumoto and K. Yoshida, Schrödinger $\sigma$-models and Jordanian twists, JHEP 08 (2013) 013 [arXiv:1305.6556] [INSPIRE].

[43] T. Kameyama and K. Yoshida, String theories on warped AdS backgrounds and integrable deformations of spin chains, JHEP 05 (2013) 146 [arXiv:1304.1286] [INSPIRE].

[44] M. Kontsevich, Deformation quantization of Poisson manifolds, Lett. Math. Phys. 66 (2003) 157 [q-alg/9709040] [INSPIRE].

[45] G. Arutyunov and S. Frolov, Foundations of the $A d S_{5} \times S^{5}$ superstring: I, J. Phys. A 42 (2009) 254003 [arXiv:0901.4937] [InSPIRE]. 